\title{
Analysis on the Application Prospect of Virtual Reality Technology and Material in Physical Education
}

\author{
QingyuWang,", Wei Meng² \\ ${ }^{1}$ Basic Department, Maanshan Technical College,Maanshan 243031, Anhui, China \\ ${ }^{2}$ Wuhan Technical College of Communications, Wuhan 430065, Hubei, China \\ *Corresponding Author.
}

\begin{abstract}
With the update and growth of science and technology, educational concepts continue to innovate. In the sports field, the use of Virtual Reality (VR) technology is undoubtedly a brand new training experience. The use of VR technology in the physical education (PE) classroom has promoted the growth of PE and also allowed students to master physical skills more comprehensively through high technology. The purpose of this article is to understand $V R$ technology, study the mterial of VR, improve the shortcomings of traditional PE through VR technology, and analyze the application prospects of VR technology in PE. This article mainly adopts the interview method, sample survey method and case analysis method to sample a survey of 120 students in three grades of a middle school to conduct questionnaire surveys and field visits to understand the students' knowledge of VR technology and the use of VR technology. We also can know the views of the PE for students. Through this series of investigations, the prospects of VR technology being applied to PE are analyzed. Experimental research results show that the most supported PE activity for VR technology at this stage is situational setting, and 35\% of students hope that VR technology will be prominent in the context of the picture. The application of VR technology in physical education will undoubtedly bring a different experience to school sports activities. However, due to the lack of funds and professionals, it is difficult to carry out VR technology in teaching activities. So we can start from the context setting and use economical means to pave the way for physical education.
\end{abstract}

Keywords:VR Technology, VR Material, Physical Education, Teaching Methods, Application Prospects

\section{Introduction}

The concept of VR technology has appeared very early, but due to the large limitations of early tools and equipment, the lack of software resources and the low demand of general users, VR technology was not valued by enterprises and training at the time. In recent years, our country's sports programs have continued to expand, and increasing the growth of training technologies has been put on the agenda, and VR technology, as an important one, has no time to delay its research and development. For improving the overall teaching level of school PE, the state emphasizes the use of VR technology to improve the quality of school high-quality PE.

Many people have conducted studies on the use of VR technology in PE. For example, Menin A believes that immersive VR can improve simulation results, increase learning gains and knowledge retention. However, it also has some limitations [1]. Vajak D said that the rapid popularity of VR devices has brought unparalleled convenience and entertainment experience to human life. Cardboard, Oculus Rift, HTC Vive and PSVR are gradually being accepted and recognized. The realization of VR informatization and remote exploration of new areas will become the focus of the use of VR technology in the education field [2]. Powell E designed and evaluated the primary PE training strategy intervention method. The intervention has a great effect on PE training and increased children's MVPA by 30\%. Qualitative data shows that in teachers' reflections on PE, mixed methods help to understand the effectiveness of interventions [3].

This article highlights the use and research of VR technology in sports training, and introduces the development of VR technology and the types of equipment characteristics. By studying the actual experimental training cases of 
VR technology and the corresponding national policy environment, the feasibility of applying VR technology in PE is proposed, and finally desktop VR technology is selected as the solution to the current PE design training. Observing the use of software as a background, the technology is reasonably integrated and applied to product design teaching courses. Finally, a desktop VR training aid was constructed and used for VR display in the classroom to better meet the subjective exploration needs of students.

\section{II.Application of Virtual Reality Technology in Physical Education}

\subsection{Characteristics of VR Technology}

It can be found in the practical use of VR technology that it has the characteristics of simulation, immersion, communication and interaction, and safety [4]. The computer can communicate with participants to avoid the dangerous problems of practical operation. VR technology can display experimental phenomena that cannot be presented in reality, visualize abstract problems, and display some less significant experimental phenomena in the form of organ sensory stimulation of listening, seeing, and seeing, and deepen the experience of the experiencer.

\subsection{Classification of VR Technology}

The desktop VR system is a small VR system based on the common PC platform. The geometric sense it brings can make the experiencer have a specific sense of involvement. The desktop VR system brings people a sense of immersion and effect. Relatively low use of funds, the application is more common. Desktop VR system is the primary stage of VR technology [5]. The immersive VR system gives the human voice an immersive feeling. Its characteristic is to isolate the experiencer's vision, hearing, and touch from the real space by wearing a specific VR device, resulting in virtual vision and tactile sensation. Distributed VR system (DVR) is in the virtual environment of the network, multiple users participate together through the computer and the network, so as to implement the interaction and sharing of information. Distributed VR system is interactive and shared [6]. The basic feature of the system reality system is to simulate reality and give people a sense of reality. The remote sensing VR system combines three-dimensional images of real objects from remote areas with virtual objects generated by computers.

\subsection{Virtual Teaching Development Environment and Tools Based on VR Technology}

Currently, the software and hardware platforms for the growth of VR systems mainly include two types of development platforms based on personal computers and workstations [7].

(1) Unreal Engine4 components. There are VR growth tools and blueprints system in Unreal Engine4. The remaining components also include graphics engine, sound effect engine, physics engine, input module, network module and script interpreter.

(2) HTC Vive operating system. HTC Vive is a VR head-mounted display product jointly developed by HTC and Valve. It can provide users with an immersive VR experience through three parts [8].

\subsection{Model Conversion}

The local coordinate system is defined as a coordinate system centered on the object. Each object has a local coordinate system. It can also be considered that each object is defined in its own object space, and multiple objects can be integrated into a world space [9]. If the form of coordinate transformation is:

ISSN: 0010-8189

$$
\begin{aligned}
& \mathrm{a}^{\prime}=x_{a a} a+x_{a b} b+x_{a c} c+y_{a} \\
& b^{\prime}=x_{b a} a+x_{b b} b+x_{b c} c+y_{b},(1) \\
& c^{\prime}=x_{c a} a+x_{c b} b+x_{c c} c+y_{c}
\end{aligned}
$$


$\mathrm{a}^{\prime}, \mathrm{b}^{\prime}$ and $\mathrm{c}^{\prime}$ are the coordinates obtained by linear transformation of the original coordinates a, b, and $\mathrm{c}$. This transformation is called Affine Transformation. Translation, rotation, scaling, reflection and miscutting are special cases of affine transformation.

In the three-dimensional homogeneous coordinate representation, the three-dimensional translation of the coordinate position can be expressed in the form of the following matrix:

$$
\left[\begin{array}{l}
\mathrm{a}^{\prime} \\
b^{\prime} \\
c^{\prime} \\
1
\end{array}\right]=\left|\begin{array}{l}
100 t_{a} \\
010 t_{b} \\
001 t_{c} \\
0001
\end{array}\right| \cdot\left[\begin{array}{l}
a \\
b \\
c \\
1
\end{array}\right](2)
$$

In the three-dimensional scene environment, the translation operation of the model object can be realized by the translation of the vertex coordinates of the model object.

For dimensional rotation operations, the corresponding rotation axis needs to be defined. First, the three-dimensional z-axis rotation equation can be obtained by formula (3):

$$
\begin{aligned}
& \mathrm{a}^{\prime}=a \cos \alpha-b \sin \alpha \\
& b^{\prime}=a \sin \alpha+b \cos \alpha(3) \\
& c^{\prime}=c
\end{aligned}
$$

Among them, $\alpha$ is the rotation angle. When the object is modeled, the object has its own local coordinate system and belongs to its own object space. In the rendering pipeline, the first task is to integrate the model objects in the independent object space into the world space.

\subsection{The Impact of VR Technology on PE}

(1) PE experiments are more acceptable. VR technology can virtualize some experimental mice in PE activities to prevent students from disgusting and nauseous to physical experiments.

(2) A change in the concept of PE. VR technology has changed the teacher-centered thinking of teaching in the past, and has improved students' autonomous participation and classroom activity [10]. VR technology can realize interdisciplinary and intercultural learning.

(3) Enriching the content of PE. The use of VR technology in PE can enrich the content of PE, enable students to learn sports in different regions and enhance their understanding of the region.

(4) Diversification of PEtraining methods. The use of VR technology has enabled teachers to diversify their PE methods for students. Not only can they use written teaching, they can also use VR technology for practical exercises [11].

(5) Timely evaluation of PEtraining. In the VR system, students learn in stages, and the system will give feedback in time for each stage of learning, and judge whether each stage of learning is qualified.

2.6 The Problems of VR Technology on PE

(1) VR products are not cost-effective. The more functions of a VR product, the higher the price. Therefore, the ISSN: 0010-8189 
school is under great financial pressure;

(2) Professionals are scarce. Due to the remoteness of some areas, the mastery of VR technology is not enough, and it is difficult to achieve successful VRtraining;

(3) The experience is not good. VR products will cause uncoordinated body movement after being worn for a long time;

(3) VR technology is imperfect. Its use in education is in its infancy, and the content is not comprehensive.So it is essential for educational workers to solve this problem first [12].

\section{III.Physical Education Teaching Experiment Based In VR Technology}

\subsection{Analysis of the Advantages of VR in PE}

(1) Set the training environment. The input and application of VR technology can set the training environment according to the needs of the course, or set the training environment according to the environment required by different projects;

(2) Choose training content. In the VR system, you can learn and practice sports skills of dangerous events, so that students can experience the different feelings and pleasures brought by different sports;

(3) Stimulate interest in learning. Apply VR technology to the PE class, mobilize the students' enthusiasm for learning, and make students full of interest in the PE class;

(4) Avoid accidents. If students learn PE in the VR system, communication between students is reduced and conflicts are avoided.

\subsection{Experimental research content}

When VR technology is applied to teaching, it not only allows students to learn knowledge, but also allows students to have a deeper impression of what they have learned. This technology can give people a real feeling through the computer. Therefore, based on the understanding of VR technology, this article conducted a questionnaire survey on the prospects of using this technology in PE. The content of the survey included whether students have knowledge of VR technology and their views on the use of VR technology in the classroom. This experiment uses a questionnaire survey method to conduct the survey, the survey is conducted online and offline at the same time.

\subsection{Experimental process}

According to the purpose of the thesis, this article designs the corresponding question content for the questionnaire survey. Send out several questionnaires in advance to make sure that the questionnaire is correct and the questions are comprehensive. After confirming the final questionnaire, the investigator is responsible for issuing and returning the questionnaire. A total of 120 questionnaires were issued, and 117 valid questionnaires were returned. The questionnaire response rate was about $97.5 \%$. This process lasts for 2 weeks. The collected questionnaires are collected and analyzed, and the survey results are obtained.

\section{IV.Experimental Analysis of Using VR Technology in Physical Education}

\subsection{Analysis of Students' Understanding of VR Technology}


VR technology is an Internet technology that has emerged in recent years. The growth of VR technology not only has a certain impact on all aspects of our lives, but the use of VR technology in the classroom makes the classroom more lively and interesting. In order to know the students' knowledge of VR technology, this article collects and analyzes the data based on the questionnaire survey and draws Table 1:

Table 1 Students' Understanding of VR Technology

\begin{tabular}{|c|c|c|c|c|c|}
\hline & Familiar & Know & Heard & Not Clear & Not Know \\
\hline Seventh Grade & 1 & 11 & 15 & 10 & 2 \\
\hline Eighth Grade & 2 & 15 & 13 & 8 & 1 \\
\hline Ninth Grade & 2 & 17 & 14 & 5 & 1 \\
\hline
\end{tabular}

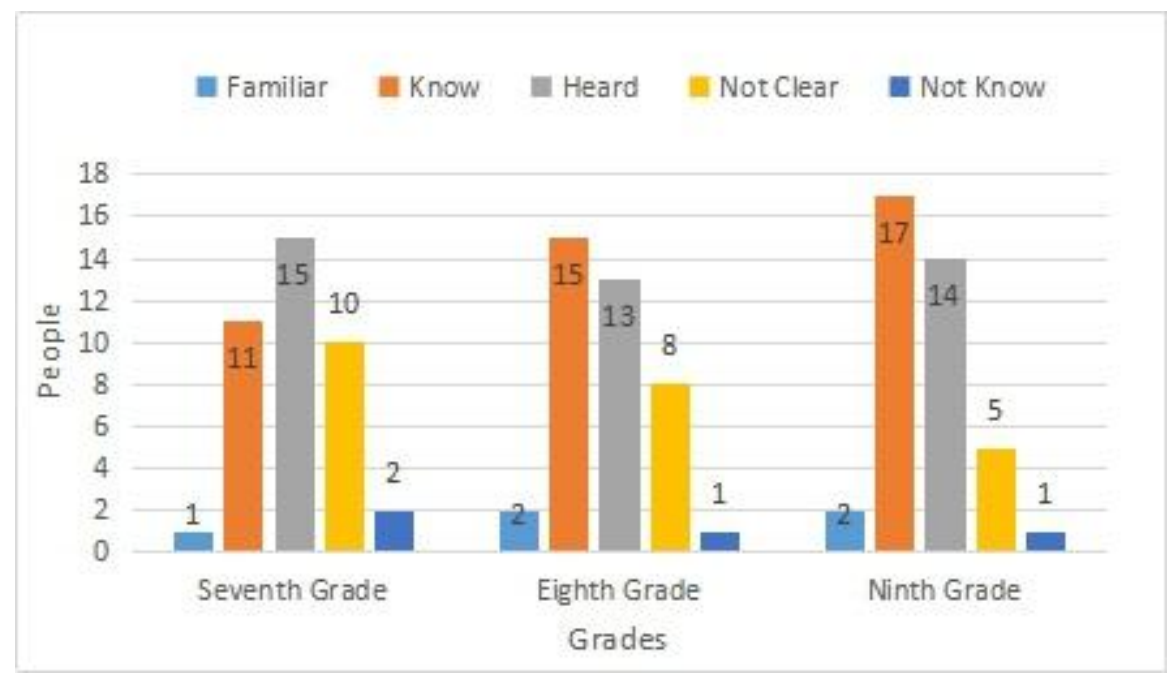

Figure 1: Students' Understanding of VR Technology

As shown in Figure 1, most of the 1 students surveyed still have some understanding of VR technology. A total of 33 of the 39 ninth grade students surveyed knew VR technology, and $85 \%$ of the students knew some VR technology. And $27 \%$ of seventh graders do not know VR technology. In summary, the higher the grade, the more knowledge about VR technology.

\subsection{Students' Discussion on Where VR Technology is used in PE Classrooms}

The content of this questionnaire involves the areas in which students hope to use VR technology, including situational setting, interaction, sports knowledge, and exercises. As shown in Table 2, more than one-third of the middle school students surveyed hope to apply VR technology in contextual settings, while a few people want to use VR technology to learn in sports knowledge training.

Table 2The Support of Four Aspects in Application of VR Technology

\begin{tabular}{|c|l|l|l|l|}
\hline & $\begin{array}{l}\text { Context } \\
\text { Setting }\end{array}$ & $\begin{array}{l}\text { Interactive } \\
\text { Session }\end{array}$ & $\begin{array}{l}\text { Sports } \\
\text { Knowledge }\end{array}$ & Practice Drill \\
\hline $\begin{array}{c}\text { Seventh } \\
\text { Grade }\end{array}$ & 15 & 10 & 7 & 7 \\
\hline Eighth Grade & 13 & 12 & 5 & 10 \\
\hline Ninth Grade & 13 & 7 & 3 & 15 \\
\hline
\end{tabular}




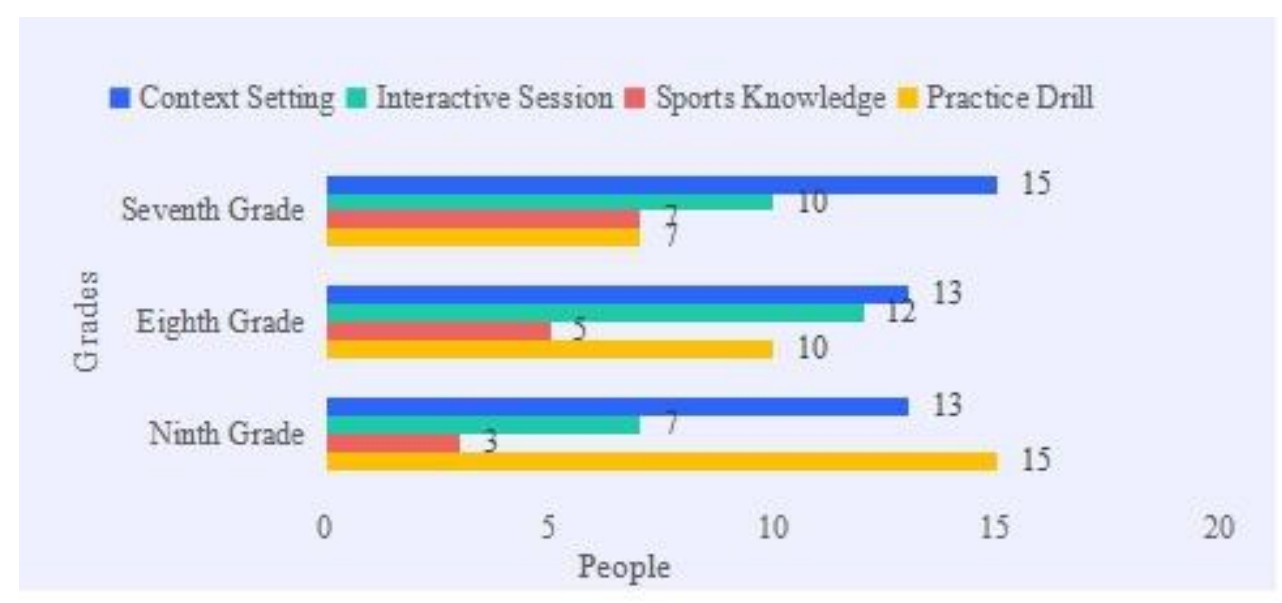

Figure 2: The Support of Four Aspects in Application of VR Technology

As shown in Figure 2, we can conclude that the current use of VR technology in contextual settings is well-received. According to the opinion of the respondents, the setting of the situation is ranked first, because the situation can make people feel more immersive, and the experience is deeper and more interesting. If the actual operation of sports events is supported by VR technology, then there will be a safer practice environment. The third place is the interactive session, and the support rate of students for interactive VR technology is not very high. At the bottom is sports knowledge. VR technology has little effect on the mastery of basic knowledge, because real knowledge comes out of practice.

\section{Conclusion}

The try of CR in training is to improve students' enthusiasm and participation. The use of VR technology in training in a reasonable way will produce good training results. In the future, VR technology will be the most critical technology in the teaching field. Although multimedia teaching has brought convenience and received unanimous praise from school, the lack of experience and old-fashioned shortcomings have prompted the use of VR technology in PE. With the maturity of VR technology, training has gradually realized virtual forms and learners' participation methods have been continuously optimized. VR technology has given full play to its own advantages and promoted the development and progress of the education field. In terms of the process of class, we must pay attention to the importance of situational setting in the use of VR to PE.

\section{Reference}

[1] Menin. A, Torchelsen. R, Nedel L. An Analysis of VR Technology Used in Immersive Simulations with a Serious Game Perspective. IEEE Computer Graphics and Applications, 2018, 38(2):57-73.

[2] Vajak. D, Livada. C, Combining Photogrammetry, 3D Modeling and Real Time Information Gathering for Highly Immersive VR Experience// Zooming Innovation in Consumer Electronics International Conference. IEEE, 2017, 32(13).82-85.

[3] Powell. E, Woodfield. L. A, Nevill A M. Increasing physical activity levels in primary school physical education: The SHARP Principles Model. Preventive Medicine Reports, 2016, 3(2).7-13.

[4] Shan. P, Sun. W. Auxiliary use and detail optimization of computer VR technology in landscape design. Arabian Journal of Geosciences, 2021, 14(9):1-14.

[5] Cynthia. H. H, Wu H. Technology for real estate education and practice: a VR technology perspective. Property Management, 2020, 38(2):311-324.

[6] Li. S, Hou. Y, Study on the Architecture Design and interior Decoration based on VR Technology and Computer Simulation Platform. Paper Asia, 2019, 35(2):54-57.

[7] Christensen, M. K. Biographical learning as health promotion in physical education. A Danish case study. European Physical Education Review, 2016, 13(1).5-24. 
[8] Ovens. A, A Quest for a Pedagogy of Critical Theorising in Physical Education Teacher Education: One Physical Educator's Journey. International Journal of Occupational Safety \& Ergonomics Jose, 2016, 19(1):87-95.

[9] Hastie. P. A, Wallhead. T, Models-Based Practice in Physical Education: The Case for Sport Education. Journal of Teaching in Physical Education, 2016, 35(4):390-399.

[10] Tolgfors. B, M Öhman. The implications of assessment for learning in physical education and health. European Physical Education Review, 2016, 22(2), 1-17.

[11] Goossens. L, Cardon. G, WitVRouw. E, et al. A multifactorial injury prevention intervention reduces injury incidence in Physical Education Teacher Education students. European Journal of Sport Science, 2016, 16(3):365-373.

[12] Adamakis. M, Zounhia. K, The Impact of Occupational Socialization on Physical Education Pre-Service Teachers' Beliefs about Four Important Curricular Outcomes: A Cross-Sectional Study. European Physical Education Review, 2016, 22(3),279-297. 